\title{
Versión argentina de la escala Monash para la evaluación de la relación de los dueños con sus perros (MDORS-AR) y análisis de los factores demográficos asociados a sus resultados
}

Argentine version of the Monash scale for the evaluation of the owners' relationship with their dogs (MDORS-AR) and analysis of the demographic factors associated with its results

\section{Agradecimientos}

Agradecemos la colaboración del Dr. Angel Elgier, director de la carrera de psicología de la Universidad Abierta Iberoamericana e investigador asistente (CONICET) y la Lic. Natalia Putrino docente de la Universidad Abierta Iberoamericana y becaria doctoral de CONICET, quienes pusieron en contacto a los autores y realizaron valiosas sugerencias metodológicas.

\section{Resumen}

La escala Monash de relación del dueño con su perro evalúa la percepción del dueño del vínculo con su perro y es ampliamente utilizada en las investigaciones del área. El objetivo del presente trabajo fue validar una versión en español de la escala con una población argentina. Para ello se administró la escala junto a una encuesta demográfica, tanto a través de internet como de manera presencial, a 1703 personas. En primer lugar, los ítems se agruparon en tres componentes principales tal como se observó en la estructura factorial de la versión original de la escala. En segundo lugar, hallamos que la forma de administración influye en los resultados ya que la personas que respondieron por internet refirieron sentirse más cercanas a sus perros y percibieron como menos costoso su mantenimiento que las que contestaron presencialmente. En tercer lugar, hubo diversas asociaciones con características demográficas: las personas que viven solas interactúan más con su perro, aquellas sin hijos reportaron una mayor interacción y cercanía emocional, los perros castrados y las hembras son percibidos como más costosos y las personas con vínculos más añejos con sus perros se sienten más cercanas

*Instituto de Ciencias Biológicas y Biomédicas del Sur (INBIOSUR - CONICET) ; Universidad Nacional del Sur (UNS). Departamento de Bilogía, Bioquímica y Farmacia, Bahia Blanca, Argentina. Mail de contacto: facarballo01@gmail.com

**Universidad Abierta Iberoamericana (UAI). Facultad de Psicología y Relaciones Humanas, Buenos Aires, Argentina

*** Consejo Nacional de Investigaciones Científicas y Técnicas. Universidad de Buenos Aires. Instituto de investigaciones Médicas (IDIM). Grupo de Investigación del Comportamiento en Cánidos (ICOC), Buenos Aires, Argentina ; Universidad de Buenos Aires. Facultad de Medicina. Instituto de investigaciones Médicas A. Lanari, Buenos Aires, Argentina Doi: https://doi.org/10.46553/RPSI.17.33.2021.p7-21

Fecha de Recepción: 2 de marzo de 2020 Fecha de Aceptación: 7 de diciembre de 2020 
emocionalmente a ellos, aunque interactúan menos. En conclusión, la presente versión de la escala resulta valida y sensible para detectar diferencias individuales respecto de la percepción de la relación de los dueños con sus perros.

Palabras clave: Vínculo humano-animal, Apego, Interacción perro-persona, Cercanía emocional, Costos percibidos de la tenencia de mascotas.

\section{Abstract}

The dogs-owners' relationship Monash scale, which evaluates the owner's perception of the bond with his dog, is a widely used tool in the studies about dog human interactions. The objective of this work was to validate a Spanish version of the scale with an Argentine population. To this end, the scale was administered together with a demographic survey, both through the Internet and face-to-face, to 1703 people. First, we found evidence that confirm the three components' factor structure of the original scale. Secondly, we found that the administration form influences the results. Since people who responded online reported being emotionally closer to their dogs and perceived its maintenance as less than those who answered in person. Third, there were several associations between dog and owner's characteristics and the scores of the scale: people who live alone interact more with their dogs, those without children reported greater interaction and emotional closeness, castrated and female dogs are perceived as more costly and people who own a dog for longer time feel emotionally closer to them even though they interact less whit their dog. In conclusion, the present version of the scale is a valid and sensitive tool to detect individual differences regarding the owners' perception of the relationship with their dogs.

Keywords: Human-animal bond,Attachment, Dog-human interaction, emotional closeness, Pet-ownership perceived costs.

\section{Introducción}

Los perros y las personas desarrollan un estrecho vínculo que se refleja en numerosos aspectos positivos de la relación. Por ejemplo, la interacción con los perros produce un aumento de sensaciones positivas, disminución de la soledad y de otras emociones negativas, mejoras en la salud y es mediador del establecimiento de nuevas relaciones sociales, entre otros beneficios (Friedmann \& Son, 2009; O'Haire, 2010; Odendaal \& Meintjes, 2003). En este sentido se vuelve crucial evaluar la percepción de las personas de las características de dicho vínculo, ya que hay enormes diferencias individuales en la forma que tenemos de relacionarnos con ellos. Una de las maneras de evaluar esta percepción es a través de cuestionarios administrados a los dueños. La escala Monash de relación del dueño con su perro (The Monash dog owner relationship scale, MDORS) desarrollada por Dwyer et al. en 2006 es una de las más utilizadas con este fin (Rehn et al., 2014). La escala cuenta con 28 ítems, y está compuesta de tres subescalas: (a) interacción dueñoperro, (b) cercanía emocional percibida y (c) costos percibidos, que representan diversos aspectos relevantes de la relación humano-perro. Es importante destacar que para la evaluación de los costos percibidos se invierten los ítems de la escala, por lo tanto, las personas que obtienen puntajes mayores en la subescala costo percibido 
sienten que es menos costoso tener un perro que aquellos que tiene puntajes bajos, esta forma de codificación permite interpretar el puntaje total de la escala como una medida general del vínculo entre el perro y su dueño. Asimismo, la versión original en inglés del cuestionario fue validado y se evaluó su fiabilidad con una muestra de 1125 dueños en Australia (Dwyer et al., 2006) y ha mostrado ser una herramienta válida y útil para su uso en el ámbito científico (Rehn \& Keeling, 2016).

En este sentido, los puntajes obtenidos en la escala MDORS, en diversos estudios, han mostrado tener asociaciones con diferentes aspectos relevantes del vínculo entre perros y personas, lo cual demuestra su pertinencia para detectar la calidad de la relación dueño-perro. Por ejemplo, van Herwijnen et al. (2018) encontraron una asociación entre la probabilidad de que los dueños estén satisfechos con la relación con sus perros con las tres subescalas de la Monash, especialmente con la percepción de los costos de poseer un perro. Asimismo, la agresión y/o la desobediencia del animal se asoció directamente con una alta percepción de los costos y una mayor probabilidad de que los dueños estén menos satisfechos con sus mascotas. En esta misma dirección, los dueños relativamente más contentos, tienden a percibir menos comportamientos no deseados en sus perros y menores costos de la tenencia del perro (Van Herwijnen et al., 2018). A su vez estos factores parecerían tener más influencia que la cercanía emocional percibida y las actividades compartidas en la satisfacción reportada de los dueños.

En la misma línea, Rehn et al. (2014) con el objetivo de estudiar la percepción de la relación de los dueños con sus mascotas, administraron el MDORS a los dueños y evaluaron a los perros en el Test de la Situación Extraña que evalúa, de forma conductual, el nivel de apego del perro al dueño. Los resultados indicaron que los dueños que interactuaban más con sus perros, medido en la subescala de interacción perro-dueño, eran aquellos que tenían perros con más comportamientos de búsqueda de proximidad y menos juego independiente en la prueba de apego.

También las características del temperamento del perro y la apariencia física parecen estar asociadas a los puntajes del MDORS. Se observó que a mayor entrenabilidad mayor percepción de cercanía emocional y menos costos percibidos. Por otro lado la belleza física o la ternura física percibida (cuteness) estuvo asociada a un mayor nivel de interacción perro-dueño (Thorn et al., 2015). Asimismo, otro estudio mostró que no solo la entrenabilidad estaría asociada con los puntajes de la MDORS sino que también los niveles de sociabilidad de los perros estarían relacionados, ya que la alta sociabilidad predijo mayores puntuaciones en la subescala de cercanía emocional (Bennett \& Rohlf, 2007).

Además, Howell et al. (2013) mostraron una asociación entre las escalas del MDORS y la percepción por parte de los dueños de las habilidades cognitivas de sus perros. Esta relación fue especialmente fuerte para la subescala de percepción de cercanía del vínculo, dado que los dueños que reportaban mayor cercanía eran los que percibían mayores habilidades cognitivas en sus perros (Howell et al., 2013).

Asimismo, la subescala de interacción perro-dueño está relacionada con la responsabilidad del dueño por la salud y el comportamiento de su perro (Rohlf et al., 2012). Aquellos dueños que más 
actividades compartían con sus perros según el MDORS eran los que más vacunaban al perro anualmente, lo llevaban a chequeos veterinarios $y$ regularmente aseaban $y$ ejercitaban a sus perros.

Un trabajo realizado en Argentina (Olarte, 2017), utilizando una versión en español no validada de la escala, halló una fuerte asociación positiva entre la cercanía emocional percibida y el nivel de antropomorfismo de los dueños. Además, a menor edad de los hijos se reportaba menor cercanía emocional y mayores costos percibidos.

Por último, la escala no sólo se ha visto que está asociada a mediciones de parámetros conductuales y cognitivos sino también a indicadores fisiológicos del vínculo, particularmente la oxitocina. Esta es una neurohormona involucrada en las conductas afiliativas, el apego y la formación de vínculos (Dzik et al., 2018). Por ejemplo, Handlin et al. (2012) hallaron correlaciones entre algunos ítems y las subescalas del MDORS con los niveles de oxitocina en perros y sus dueñas. Específicamente, a mayores niveles de oxitocina en los perros se observó una mayor frecuencia de ser besado y menor frecuencia de recibir recompensas de comida de los dueños. Más aún, los niveles más altos de oxitocina en los perros también se asociaron a menores costos percibidos. Respecto de los dueños se halló una correlación positiva entre los niveles de oxitocina y la frecuencia de besar a sus perros (Handlin et al., 2012).

Estos trabajos en su conjunto muestran que la escala mide aspectos asociados a características relevantes del vínculo entre el perro y su dueño. Pese a este hecho y a la relevancia de contar con mediciones estandarizadas de dicho vínculo, hasta nuestro conocimiento solo hay un trabajo que valida una versión del MDORS-M en idioma español realizado en México (González-Ramírez et al., 2017). Es importante destacar que una limitación de este trabajo es que solo se administró la escala por internet, lo que podría sesgar la muestra y los resultados obtenidos (Grandcolas \& Marusenko, 2003).

El objetivo del presente estudio es entonces realizar una validación de la escala MDORS en una población argentina. A su vez, se propone evaluar si la forma de administración — presencial vs internetinfluye en los resultados. Dado que se ha observado que las encuestas realizadas a través de la web aportan datos de menos calidad que las administradas de manera persona (Bowling, 2005; Couper, 2000). Por ejemplo, se incrementa el número de norespuestas, la discriminación en el ranking de ítems de escala es menor y aumentan las respuestas de tipo "no sabe/no contesta" (Heerwegh \& Loosveldt, 2008). Por último, se buscó investigar si existe una asociación entre las variables demográficas - sexo, edad, estado civil, composición familiar y características del perro-y los puntajes de la escala ya que antecedentes previos sugieren la existencia de dichas asociaciones (Diaz Videla \& Olarte, 2016; 2017). Este trabajo no solo aportará una herramienta valiosa que permitirá unificar su uso en nuestro medio, sino que favorecerá el desarrollo de nuevas investigaciones regionales en esta temática.

\section{Materiales y métodos}

\section{Declaración ética}

El estudio fue realizado de acuerdo a los 
principios éticos mencionados en la APA y en el Código Deontológico y Bioético del Ejercicio de la Psicología en Argentina relacionados con la investigación $\mathrm{y}$ publicación de información. Asimismo, todos los participantes dieron su consentimiento informado para participar de esta investigación.

\section{Participantes}

Se tomaron un total de 1703 cuestionarios los cuales iban acompañados de una breve encuesta socio-demográfica ad hoc. El $90 \%$ de los cuestionarios fueron completados por internet. La difusión se realizó a través de las redes sociales y en las páginas webs de los institutos de investigación involucrados $(\mathrm{n}=$ 1557 ; edad $=33.93 \pm 10.32 ; 1309$ Mujeres y 238 Varones). Asimismo 147 (edad $=$ $39.46 \pm 14.72$; 93 mujeres y 54 varones) cuestionarios fueron completados en papel. Los cuestionaros fueron entregados a los participantes y recolectados luego de una semana aproximadamente. La muestra total quedo compuesta por 1402 mujeres, 292 varones de entre 19 y 78 años de edad $($ media $=34.40 \pm 10.88)$. El $43 \%$ de los participantes era soltero, el $50 \%$ estaba casado o en convivencia al momento de la encuesta y el resto era viudo o divorciado/ separado. Todos los participantes dieron su consentimiento informado al momento de realizar la encuesta.

\section{Instrumentos}

La versión completa de la encuesta se puede encontrar en la Tabla 1 y en el siguiente link (https://goo.gl/forms/ HeWH99ocTsUyBDgH2). La versión argentina de la escala se basó en la traducción mejicana utilizada por González-
Ramírez et al., 2017. Primero, se realizaron pequeñas modificaciones para hacer sus enunciados más acordes a las formas regionales argentinas, por ejemplo, el enunciado: "¿Con que frecuencia llevas a tu perro en tu carro?" fue remplazado por: “¿Con que frecuencia llevás a tu perro en tu auto?". Luego se administró la escala a un grupo de voluntarios para evaluar si los nuevos enunciados eran comprensibles y se realizaron las modificaciones necesarias.

\section{Procedimiento}

Para analizar los datos, en primer lugar, se exploraron las respuestas obtenidas para investigar si las respuestas de cada uno de los ítems presentaban sesgo onula varianza, loque podría indicar que no serían relevantes para el análisis factorial. Luego, se procedió con un análisis factorial exploratorio utilizando los 28 ítems originalmente propuestos. En este primer análisis se utilizó el método de componentes principales y los resultados fueron rotados utilizando la rotación Varimax para simplificar la interpretación de los resultados. Luego se evaluó la consistencia interna de cada subescala y de la escala total usando el coeficiente de correlación alfa de Cronbach y se indago si las subescalas estaban correlacionadas con el coeficiente de correlación de Pearson. Asimismo, se realizó un análisis confirmatorio (SEM) para evaluar la adecuación del modelo original de tres factores propuesto por Dwyane et al. (2006). Todos los análisis fueron realizados utilizando el paquete estadístico SPSS v.22 y Stata 13.

Porúltimo, se calcularon los puntajes para cada subescala y para la escala total y se investigó si la forma de administración y las variables sociodemográficas exploradas 
influían en los resultados observados, utilizando la prueba $t$ para comparaciones entre grupos y el coeficiente de correlación Pearson para las variables continuas. Solo se reportan los resultados positivos. Todas las pruebas fueron a dos colas con $\alpha=0.05$.

\section{Resultados}

La exploración de los datos mostró que los ítems 8 (¿Con qué frecuencia tu perro esta con vos cuando te relajas?) y 19 (¿Qué tan doloroso crees que será para vos cuando tu perro muera?) presentaban una distribución sesgada hacia los valores positivos (Mediana $=5 ; \mathrm{Q} 1=5 ; \mathrm{Q} 3=5)$ y relativamente poca varianza (Item 8: media $=4.68, \mathrm{SD}=0.75$; Item 19: media $=4.84, \mathrm{SD}=0.43$ ).

Los estadísticos descriptivos mostraron que la muestra era adecuada para el análisis factorial $(\mathrm{KMO}=0.89$, test de esfericidad de Barlett $p<0.001$ ). Se encontraron 5 componentes principales con autovalores mayores a 1 los cuales explican el $54.92 \%$ de la varianza total de la muestra. La tabla 1 muestra los autovalores y el porcentaje de varianza explicado por cada factor en nuestro estudio y los valores reportados en González-Ramírez et al. (2017). En nuestro estudio con los primeros tres componentes se explica el $45.8 \%$ de la varianza, un resultado similar al de González-Ramírez et al. (2017) quienes encontraron que la solución de 3 factores explicaba el $48.4 \%$ de la varianza de su muestra. Si se considera un cuarto factor, el porcentaje de varianza total explicada se incrementa solo en un $4.71 \%$. Por lo que la solución factorial de 3 factores parece ser la más satisfactoria. Por lo tanto, restringimos la solución a tres factores y volvimos a realizar el análisis factorial. La tabla 2 muestra los pesos factoriales mayores a 0.4 (Tabachnik \& Fidell, 2013) de la matriz rotada en los tres factores seleccionados.

Removiendo los ítems 8 y 19 encontramos un resultado similar ya que con 3 factores logramos explicar el 46.99\% de la varianza total, por lo tanto, habría que considerar remover estos ítems de la escala.

\section{Tabla 1}

Autovalores y el porcentaje de varianza explicado por cada factor en el presente estudio y González-Ramírez et al. (2017)

\begin{tabular}{lllllll}
\hline CP & \multicolumn{3}{c}{ Carballo et al. (2019) } & \multicolumn{3}{c}{ González-Ramírez et al. (2017) } \\
\cline { 2 - 7 } & Autovalor & $\begin{array}{l}\text { Varia n z a } \\
\text { explicad a } \\
(\%)\end{array}$ & $\begin{array}{l}\text { Varianza } \\
\text { explicada } \\
\text { acumulada } \\
(\%)\end{array}$ & $\begin{array}{l}\text { Autovalor } \\
\text { Varianza }\end{array}$ & $\begin{array}{l}\text { Varianza } \\
\text { explicada } \\
\text { (\%) }\end{array}$ & $\begin{array}{l}\text { acumulada } \\
(\%)\end{array}$ \\
\hline 1 & 7.37 & 26.33 & 26.33 & 7.32 & 26.2 & 26.2 \\
2 & 3.44 & 12.31 & 38.65 & 3.22 & 11.5 & 37.7 \\
3 & 2.00 & 7.15 & 45.80 & 3.002 & 10.7 & 48.4 \\
4 & 1.31 & 4.711 & 50.51 & & & \\
5 & 1.23 & 4.40 & 54.92 & & & \\
\hline
\end{tabular}




\section{Tabla 2}

Items de la escala y carga en cada uno de los componentes.

\begin{tabular}{|c|c|c|c|}
\hline \multirow[t]{2}{*}{ Items } & \multicolumn{3}{|c|}{ Componente } \\
\hline & 1 & 2 & 3 \\
\hline 1. ¿Con qué frecuencia jugás con tu perro? & & & ,609 \\
\hline $\begin{array}{l}\text { 2. ¿Con qué frecuencia tu perro te acompaña cuando } \\
\text { visitas a otras personas? }\end{array}$ & & & ,713 \\
\hline $\begin{array}{l}\text { 3. ¿Con qué frecuencia le das premios de comida a tu } \\
\text { perro? }\end{array}$ & & & ,495 \\
\hline 4. ¿Con qué frecuencia le das besos a tu perro? & ,412 & &, 519 \\
\hline 5. ¿Con que frecuencia llevás a tu perro en tu auto? & & &, 716 \\
\hline 6. ¿Con que frecuencia abrazás a tu perro? & & &, 581 \\
\hline $\begin{array}{l}\text { 7. ¿Con que frecuencia comprás cosas para tu perro } \\
\text { (regalos, juguetes, etc)? }\end{array}$ & & &, 569 \\
\hline $\begin{array}{l}\text { 8. ¿Con que frecuencia tu perro esta con vos cuando te } \\
\text { relajas (por ejemplo, mientras ves televisión)? }\end{array}$ & & &, 510 \\
\hline 9. ¿Con que frecuencia cepillás a tu perro? & & &, 507 \\
\hline 10. Mi perro me ayuda en tiempos difíciles & ,719 & & \\
\hline 11. Mi perro siempre está para mí cuando necesito consuelo & ,762 & & \\
\hline 12. Quisiera que mi perro estuviera conmigo todo el tiempo & ,732 & & \\
\hline 13. Mi perro me brinda compañía constante & ,738 & & \\
\hline $\begin{array}{l}\text { 14. Si todos me abandonaran, mi perro estaría ahí para mí } \\
\text { (contaría con mi perro) }\end{array}$ & ,805 & & \\
\hline 15. Mi perro me da una razón para levantarme en la mañana & ,713 & & \\
\hline $\begin{array}{l}\text { 16. Quisiera que mi perro y yo nunca tuviéramos que } \\
\text { separarnos }\end{array}$ & ,757 & & \\
\hline $\begin{array}{l}\text { 17. Mi perro está constantemente pendiente de mi (me } \\
\text { presta atención) }\end{array}$ &, 538 & & \\
\hline $\begin{array}{l}\text { 18. ¿Con que frecuencia le decís cosas a tu perro que no le } \\
\text { decís a nadie más? }\end{array}$ & ,610 & & \\
\hline $\begin{array}{l}\text { 19. ¿Qué tan doloroso crees que será para vos cuando tu } \\
\text { perro muera? }\end{array}$ &, 527 & & \\
\hline
\end{tabular}

Continua en página 14

Revista de Psicología. Año 2021. Vol. 17, N 33, pp. 7-21 
Continua en página 13

\begin{tabular}{|c|c|c|c|}
\hline \multirow[t]{2}{*}{ Items } & \multicolumn{3}{|c|}{ Componente } \\
\hline & 1 & 2 & 3 \\
\hline $\begin{array}{l}\text { 20. ¿Con que frecuencia sentís que cuidar a tu perro es un } \\
\text { trabajo difícil? }\end{array}$ & & ,725 & \\
\hline $\begin{array}{l}\text { 21. Es molesto que algunas veces tengo que cambiar mis } \\
\text { planes debido a mi perro }\end{array}$ & & ,752 & \\
\hline $\begin{array}{l}\text { 22. Me molesta que por mi perro dejé de hacer cosas que } \\
\text { disfrutaba haciendo antes de tenerlo }\end{array}$ & & ,718 & \\
\hline $\begin{array}{l}\text { 23. Hay cosas importantes relacionadas a tener un perro que } \\
\text { no me gustan }\end{array}$ & & ,682 & \\
\hline $\begin{array}{l}\text { 24. ¿Con que frecuencia tu perro te limita de hacer cosas } \\
\text { que querés hacer? }\end{array}$ & & ,706 & \\
\hline 25. Mi perro hace mucho desorden & &, 517 & \\
\hline 26. Gasto mucho dinero en mi perro & &, 509 & \\
\hline 27. ¿Qué tan difícil es cuidar a tu perro? & & ,678 & \\
\hline $\begin{array}{l}\text { 28. ¿Con que frecuencia sentís que tener un perro es más un } \\
\text { costo que un beneficio? }\end{array}$ & & ,411 & \\
\hline
\end{tabular}
Nota: sólo se reportan aquellos valores mayores a 0.4 .

Los ítems concuerdan con lo esperado en función de los trabajos previos (Dwyer et al., 2006; González-Ramírez et al., 2017). Sin embargo, el ítem 4 ( $-_{i}$ Con qué frecuencia besás a tu perro? - tiene una carga mayor a 0.4 en dos de los componentes esperados.

A continuación, evaluamos la consistencia interna de cada subescala y de la escala total. El alfa de Cronbach para la subescala interacción dueño-perro fue de 0.79; para la subescala cercanía emocional percibida de 0.88 y para la de costo percibido fue de 0.81 . El alfa de Cronbach para la escala total fue de 0.79. Removiendo el ítem 4 de la subescala de cercanía emocional percibida el alfa de Cronbach de dicha subescala se incrementa un 0.03 (0.91) dado que este inciso carga positivamente en dos de las subescalas y su remoción incrementa la consistencia interna de la subescala cercanía emocional percibida, se debería considerar remover este ítem de la escala.

En línea con lo anterior encontramos que las escalas interacción dueño perro y cercanía emocional percibida estaban correlacionadas $(r=0.52, p<0.001)$ y que la subescala costo percibido correlaciona negativamente tanto con la escala interacción dueño perro $(r=-0.21, p<0.001)$ como con la escala cercanía emocional $(r=-0.18$, $p<0.01)$. Estos resultados confirman los hallazgos de González-Ramírez et al. (2017) y de Dwyane et al. (2015).

Nuevamente, si se remueven los ítems 4,8 y 19 el análisis factorial arroja 
resultados similares a los obtenidos con la escala completa. Con tres componentes se logra explicar el $47.94 \%$ de la varianza y los ítems cargan en los mismos factores.

Por último, realizamos el análisis confirmatorio del modelo estimado de 3 componentes correlacionados ente sí y con los ítems agrupados de acuerdo al modelo original y correlacionados dentro de cada subescala. Todos los parámetros fueron significativos $(\mathrm{X} 2 / \mathrm{gl}=2.38, p<0.01$; SRMR $=0.02 ;$ RMSEA $=0.03 ; \mathrm{CFI}=0.97 ; \mathrm{TLI}=$ 0.96) y se observó un buen ajuste general (Schmitt, 2011).

Con el fin de evaluar la validez externa de la escala al final del cuestionario se le pregunto a los participantes si volverían a tener un perro; asumiendo que aquellos que respondiesen afirmativamente tendrían una relación más satisfactoria que los que respondiesen de forma negativa. Solo el $3.4 \%$ de las personas reportaron que no volverían a tener un perro. Estas personas obtuvieron puntajes significativamente menores en las subescalas interacción dueño-perro, $t(1658)=8.67, p<0.001$, y cercanía emocional percibida, $t(1677)=$ 9.77, $p<0.001$; un puntaje mayor en la escala costos percibido, $t(1696)=-8.8, p=0.003$, y concomitantemente un puntaje menor en la escala global, $t(1641)=6.05, p<0.001$.

Efectos de la forma de administración y las variables socio-demográficas en los puntajes obtenidos en la escala

La tabla 3 muestra la media y el error estándar de los puntajes de la escala en función de las diferentes variables demográficas evaluadas. Las personas que respondieron por internet obtuvieron puntajes más altos en la escala total, así como en las subescalas cercanía emocional y costo percibido. No se observaron diferencias en la subescala interacción dueño-perro. Asimismo, las mujeres puntuaron más alto en la escala global $\mathrm{y}$ en las subescalas; cercanía emocional e interacción dueño perro y más bajo en costo percibido.

Con respecto a la vivienda y la composición familiar, las personas que viven solas reportan tener más interacción con sus perros que las que viven acompañadas y asimismo puntuaron más alto en la escala total. En el mismo sentido, las personas sin hijos interactúan más con sus perros y reportan mayor cercanía emocional que aquellos que tiene hijos. Consecuentemente, obtuvieron puntajes más altos en la escala total.

En lo que concierne a las características del perro los perros machos les insumen menos costos a los dueños. Asimismo, los perros castrados - machos o hembras - son percibidos como más costosos. De modo similar, los dueños con perros castrados se sienten más cercanos a sus perros que los dueños de perros sin castrar.

Por otro lado, se encontró una correlación significativa, positiva y baja entre la edad del dueño y la interacción con el perro $(r=0.054, p=0.02)$. Del mismo modo, se observó una correlación negativa entre la edad del perro y la interacción perrodueño $(r=-10, p<0.001)$ y el puntaje total de la escala $(r=0.056, p=0.02)$.

Asimismo, el tiempo desde que la persona tiene a su perro influyó en los resultados de la escala. Los dueños que tienen a sus perros desde hace más tiempo interactúan menos con ellos $(r=$ -0.064, $p=0.009)$, se sienten más cercanos 


\section{Tabla 3}

Media y error estándar de los puntajes obtenidos en la escala total y en las sub-escalas en función de las diferentes variables socio-demográficas obtenidas.

\begin{tabular}{|c|c|c|c|c|}
\hline Sub-Escala & \multicolumn{2}{|c|}{ Puntaje Según Variable } & $t(\mathrm{gl})$ & $p$ \\
\hline & \multicolumn{2}{|c|}{ Administración } & & \\
\hline & Internet & Papel & & \\
\hline Interacción Dueño - perro & $30.38(0.15)$ & $30.13(0.82)$ & $t(1662)=0.46$ & 0.64 \\
\hline Cercanía Emocional & $43.07(0.16)$ & $39.91(0.60)$ & $t(1682)=5.67$ & $<0.001$ \\
\hline Costo Percibido & $19.77(0.14)$ & $17.53(0.51)$ & $t(1702)=4.61$ & $<0.001$ \\
\hline \multirow[t]{3}{*}{ Puntaje total } & $93.34(0.28)$ & $87.54(1.11)$ & $t(1645)=5.98$ & $<0.001$ \\
\hline & \multicolumn{2}{|c|}{ Sexo de los dueños } & & \\
\hline & Mujeres & Varones & & \\
\hline Interacción Dueño - perro & $30.65(0.16)$ & $28.92(0.43)$ & $t(1652)=4.19$ & $<0.001$ \\
\hline Cercanía Emocional & $43.37(0.16)$ & $40.08(0.43)$ & $t(1672)=8.00$ & $<0.001$ \\
\hline Costo Percibido & $19.47(0.14)$ & $20.26(0.34)$ & $t(1692)=-2.17$ & 0.03 \\
\hline \multirow[t]{3}{*}{ Puntaje total } & $93.53(0.29)$ & $89.55(0.74)$ & $t(1635)=5.41$ & $<0.001$ \\
\hline & \multicolumn{2}{|c|}{ Vive solo } & & \\
\hline & $\mathrm{Si}$ & No & & \\
\hline Interacción Dueño - perro & $31.84(0.36)$ & $30.06(0.16)$ & $t(1608)=3.90$ & $<0.001$ \\
\hline Cercanía Emocional & $43.18(0.42)$ & $42.77(0.17)$ & $t(1629)=0.86$ & 0.38 \\
\hline Costo Percibido & $19.42(0.37)$ & $19.73(0.14)$ & $t(1648)=-0.77$ & 0.44 \\
\hline \multirow[t]{3}{*}{ Puntaje total } & $94.63(0.71)$ & $92.65(0.30)$ & $t(1592)=2.38$ & 0.01 \\
\hline & \multicolumn{2}{|c|}{ Tiene hijos } & & \\
\hline & $\mathrm{Si}$ & No & & \\
\hline Interacción Dueño - perro & $29.13(0.34)$ & $30.79(0.17)$ & $t(1653)=4.71$ & $<0.001$ \\
\hline Cercanía Emocional & $42.19(0.33)$ & $42.99(0.17)$ & $t(1673)=2.23$ & 0.02 \\
\hline Costo Percibido & $19.70(0.28)$ & $19.55(0.15)$ & $t(1693)=-0.47$ & 0.63 \\
\hline \multirow[t]{3}{*}{ Puntaje total } & $91.12(0.57)$ & $93.42(0.31)$ & $t(1636)=3.63$ & $<0.01$ \\
\hline & \multicolumn{2}{|c|}{ Sexo del perro } & & \\
\hline & Macho & Hembra & & \\
\hline Interacción Dueño - perro & $30.15(0.22)$ & $30.53(0.21)$ & $t(1657)=-1.20$ & 0.22 \\
\hline Cercanía Emocional & $42.76(0.23)$ & $42.81(0.21)$ & $t(1677)=-0.15$ & 0.87 \\
\hline Costo Percibido & $20.11(0.20)$ & $19.14(0.18)$ & $t(1697)=3.52$ & $<0.001$ \\
\hline \multirow[t]{3}{*}{ Puntaje total } & $93.04(0.40)$ & $92.64(0.38)$ & $t(1640)=0.46$ & 0.46 \\
\hline & \multicolumn{2}{|c|}{ Estado reproductivo } & & \\
\hline & Castrado & Entero & & \\
\hline Interacción Dueño - perro & $30.62(0.20)$ & $30.00(0.24)$ & $t(1656)=1.99$ & 0.46 \\
\hline Cercanía Emocional & $43.28(0.19)$ & $42.21(0.25)$ & $t(1676)=3.37$ & 0.001 \\
\hline Costo Percibido & $19.20(0.17)$ & $20.06(0.21)$ & $t(1696)=-3.13$ & 0.002 \\
\hline Puntaje total & $93.22(0.35)$ & $92.34(0.44)$ & $t(1639)=1.57$ & 0.11 \\
\hline
\end{tabular}


emocionalmente $(r=0.064, p=0.009)$ $\mathrm{y}$ sienten que es más costoso mantener a su perro $(r=-0.12 p<0.001)$. Además, el tiempo desde que los dueños viven con su perro correlacionó negativamente con el puntaje total de la escala $(r=-0.062, p=$ $0.01)$.

\section{Discusión}

El objetivo de este trabajo fue, en primer lugar, validar una versión argentina de la escala MDORS que evalúa la percepción de los dueños de su vínculo con sus perros. Los resultados obtenidos son similares a los de la validación de la encuesta en una población mejicana (González-Ramírez et al., 2017) y a los de la escala original (Dwyer et al., 2006). Los ítems se agruparon en tres componentes principales de un modo análogo a las investigaciones previas, ratificando la estructura factorial propuesta originalmente.

Sin embargo, se podrían realizar algunas mejoras en la escala. En primer lugar, hemos encontrado que el ítem 4 “ ¿Con qué frecuencia le das besos a tu perro?" carga en dos factores "interacción dueñoperro" y "cercanía emocional percibida". Dado que el puntaje de este ítem colabora a la discriminación de los tipos de vínculos de los dueños y sus perros, una posibilidad sería sumar la puntuación de este ítem para obtener el puntaje total de las dos subescalas. Aunque dado que, en coincidencia con la escala original, este inciso tiene una mayor carga en el componente "interacción dueño-perro" creemos que para hacer más comparables los resultados obtenidos es mejor mantener la codificación original. Asimismo, al analizar la consistencia de cada subescala con y sin el ítem 4 encontramos que si eliminamos el inciso de la subescala "cercanía emocional percibida" se incrementa su consistencia interna apoyando lo antedicho.

Por otro lado, los ítems 8: “¿Con que frecuencia tu perro esta con vos cuando te relajas (por ejemplo, mientras ves televisión)?” y 19 “¿Qué tan doloroso crees que será para vos cuando tu perro muera?" mostraron poca varianza siendo poco informativos para el análisis factorial (Sheppard \& Mills, 2002), por lo que se debe considerar su remoción de la escala.

Finalmente, incluimos en nuestro cuestionario una pregunta para evaluar la validez externa de la escala, asumiendo que aquellas personas con una relación más satisfactoria con su mascota estarían más dispuestas a volver a elegir tener un perro. Interesantemente, las personas que respondieron que no volverían a tener un perro tuvieron puntajes menores en las subescalas "cercanía emocional" e "interacción dueño-perro", un puntaje mayor en la subescala "costo percibido" y un puntaje menor en la escala general. Por lo tanto, la escala pudo captar las diferencias individuales en los vínculos entre diferentes dueños con sus perros, mostrando la validez externa de la misma.

En suma, la MDORS-AR sería un elemento útil y valido para investigar el vínculo entre los dueños y sus mascotas. Futuras investigaciones podrían desarrollar una versión mejorada de la escala, teniendo en cuenta estos resultados.

En segundo lugar, en el presente estudio nos propusimos evaluar si la forma de administración de la escala influía en los resultados. Se encontró que las personas que completaron la escala por internet refirieron sentirse más cercanas a sus perros y que el 
mantenimiento de su mascota era menos costoso que aquellos que respondieron a las encuestas impresas en papel. Por lo tanto, la forma de administración influyó en las percepciones de las personas respecto al vínculo con su mascota. Sin embargo, es interesante destacar que no encontramos diferencias en la subescala "interacción dueño-perro" que indaga sobre la frecuencia de conductas específicas que las personas llevan a cabo con sus animales. Por lo tanto, en el desarrollo de futuras escalas sería conveniente introducir más preguntas que hagan referencia a los hábitos que los dueños muestran con sus perros más que sobre las expectativas subjetivas de las personas con respecto a su relación con sus perros, dado que estas apreciaciones son más propensas a ser sesgadas.

En tercer lugar, el objetivo fue evaluar la relación entre la escala y ciertas características demográficas de la población. Hallamos que las personas que viven solas interactúan más con su perro que aquellas que viven acompañadas. Esto concuerda con datos previos que muestran que las personas que viven solas también brindan más cuidado a sus perros (Dotson \& Hyatt, 2008). Posiblemente, las personas que viven solas desarrollen un vínculo más estrecho con sus mascotas, las cuales son percibidas como una compañía y una fuente de confort (e.g. O'Haire, 2010; Polheber \& Matchock, 2013) y con quienes forman fuertes vínculos de apego (Odendaal \& Meintjes, 2003).

En la misma línea, las personas sin hijos reportaron una mayor interacción con sus perros y refirieron sentirse más cerca emocionalmente que aquellas con hijos. Estos datos concuerdan con Meyer y Forkman (2014) que utilizando la escala original encontraron el mismo resultado. Posiblemente las personas sin hijos tengan más tiempo para interactuar con sus perros y que esto genere un sentimiento de mayor cercanía. Asimismo, nuestros datos aportan evidencia a la idea de que la presencia de niños en el hogar influye negativamente en el vínculo entre los perros y las personas como sugieren varios estudios previos (Bennett \& Rohlf, 2007; Dotson \& Hyatt, 2008; Marinelli et al., 2007). De ser así, es importante señalar que la escala utilizada (MDORS-AR) fue lo suficientemente sensible como para detectar esta diferencia.

Con respecto a cómo las características de los perros influyen en el vínculo, por un lado, encontramos que los perros castrados son percibidos como más costosos. Es factible que los dueños que castran a sus perros sean más responsables en su tenencia, lo que recae en un costo mayor de mantenimiento de los perros. Sin embargo, este resultado no concuerda con los estudios previos realizados en el país que no encuentran efectos del estado reproductivo del animal en ninguna de las subescalas de la MDORS (Diaz Videla \& Olarte; 2017). Posiblemente, esta diferencia se deba a las divergencias metodológicas entre los estudios (modalidad de muestreo, tamaño de la muestra y herramientas utilizadas ya que en Diaz Videla \& Olarte, 2017 se utiliza una versión modificada de la escala MDORS). Por otro lado, las hembras son percibidas como más costosas que los machos. Si bien este resultado va en contra de lo esperado, dado que por un lado no se han encontrado diferencias de sexo en el vínculo entre perros y personas en un estudio similar (Diaz Videla \& Olarte, 2017) y por el otro lado a que los machos suelen ser más audaces y agresivos 
que las hembras (Scandurra et al., 2017), lo que podría conllevar a mayores problemas de conducta que podrían repercutir negativamente en el vínculo (Bennett \& Rohlf, 2007). Sin embargo, en este estudio no encontramos que esto represente un costo especial para los dueños. Se deberían realizar más investigaciones para determinar qué características distintivas de los machos y las hembras influyen en el costo percibido.

Por último, en línea con previo estudios, hemos observado evidencias de que el vínculo entre los perros y sus dueños va desarrollándose y cambiando con el tiempo dado que encontramos correlaciones bajas pero significativas entre los puntajes de la escala y el tiempo desde que la personas tienen a sus perros, así como con la edad tanto del dueño como de la mascota (Dotson \& Hyatt, 2008; Marinelli et al., 2007; Diaz
Videla \& Olarte, 2017). Es importante notar que las personas con vínculos más añejos con sus perros se sienten más cercanas emocionalmente a ellos, aunque interactúan menos. Esto podría estar relacionado con el hecho de que los perros de mayor edad suelen tener menor nivel de actividad. Es decir, estas correlaciones muestran que ambos miembros de la diada dueño-perro participan y colaboran en la construcción de un vínculo cada vez más cercano entre ellos.

En síntesis, podemos concluir que la escala MDORS-AR es una herramienta válida y suficientemente sensible como para captar diferencias en el tipo de vínculo entre los perros y sus dueños. Consideramos que su utilización puede aportar al avance en el estudio científico de los vínculos entre perros y personas.

\section{Referencias}

Bennett, P. C., \& Rohlf, V. I. (2007). Ownercompanion dog interactions: Relationshipsbetween demographic variables, potentially problematic behaviours, training engagement and shared activities. Applied Animal Behaviour Science, 102(12), 65-84. https://doi.org/10.1016/j. applanim.2006.03.009

Bowling, A. (2005). Mode of questionnaire administration can have serious effects on data quality. Journal of Public Health, 27(3), 281-291. https://doi.org/10.1093/pubmed/ fdi031

Couper, M. P. (2000). Web surveys: A review of issues and aproaches. Public Opinion Quarterly at University of Georgia On, 64, 464-494.
Diaz Videla, M., \& Olarte, M. A. (2017). Dogs 'Demographic Characteristics Associated with Relationship Differences Perceived by the Guardian, European Scientific Journal. 6, 218-232.

Diaz Videla, M., \& Olarte, M. A. (2016). Animales de compañía, personalidad humana

y los beneficios percibidos por los custodios. PSIENCIA Revista latinoamericana de ciencia psicológica, 8(2), 1-19. https://doi.org/10.5872/ psiencia/8.2.21

Dotson, M. J., \& Hyatt, E. M. (2008). Understanding dog-human companionship. Journal of Business Research, 61(5), 457466. https://doi.org/10.1016/j. 
jbusres.2007.07.019

Dwyer, F., Bennett, P. C., \& Coleman, G. J. (2006). Development of the Monash Dog Owner Relationship Scale (MDORS). Anthrozoos, 19(3), 243-256. https://doi. org $/ 10.2752 / 089279306785415592$

Dzik, V., Barrera, G. \& Bentosela, M. (2018) La importancia de la oxitocina en el vínculo entre perros y personas. Interdisciplinaria. Revista de Psicología y Ciencias Afines. Interdisciplinaria, 35[2], 527-542.

Friedmann, E., \& Son, H. (2009). The Human-Companion Animal Bond: How Humans Benefit. Veterinary Clinics of North America - Small Animal Practice, 39(2), 293326. https://doi.org/10.1016/j. cvsm.2008.10.015

González-Ramírez, M. T., Vanegas-Farfano, M., \& Landero-Hernández, R. (2017). Versión mexicana de la escala Monash de relación del dueño con su perro (MDORSM). Alternativas En Psicología, 107-123. Retrieved from http:// www.alternativas.me/26-numero37-febrero-julio-2017/149-versionmexicana-de-la-escala-monash-derelacion-del-dueno-con-su-perromdors-m

Grandcolas, U., And, R. R., \& Marusenko, K. (2003). Ursula Grandcolas , Ruth Rettiei and Kira Marusenko Web Survey Bias : Sample or Mode Effect? Journal of Marketing Management, 19(Couper 2000), 541-561.

Handlin, L., Nilsson, A., Ejdebäck, M., Hydbring-Sandberg, E., \& Uvnäs-
Moberg, K. (2012). Associations between the psychological characteristics of the human-dog relationship and oxytocin and cortisol levels. Anthrozoos, 25(2), 215-228. https://doi.org/10.2752/1 75303712X13316289505468

Heerwegh, D., \& Loosveldt, G. (2008). Faceto-face versus web surveying in a high-internet-coverage population: Differences in response quality. Public Opinion Quarterly, 72(5), 836-846. https://doi.org/10.1093/ poq/nfn045

Howell, T. J., Toukhsati, S., Conduit, R., \& Bennett, P. (2013). Do dogs use a mirror to find hidden food? Journal of Veterinary Behavior: Clinical Applications and Research, 8(6), 425-430. https://doi.org/10.1016/j. jveb.2013.07.002

Marinelli, L., Adamelli, S., Normando, S., \& Bono, G. (2007). Quality of life of the pet dog: Influence of owner and dog's characteristics. Applied Animal Behaviour Science, 108(1-2), 143-156. https://doi.org/10.1016/j. applanim.2006.11.018

O'Haire, M. (2010). Companion animals and human health: Benefits, challenges, and the road ahead. Journal of Veterinary Behavior: Clinical Applications and Research, 5(5), 226-234. https://doi.org/10.1016/j. jveb.2010.02.002

Odendaal, J. S. J., \& Meintjes, R. A. (2003). Neurophysiological correlates of affiliative behaviour between humans and dogs. Veterinary Journal, 165(3), 296-301. 
Versión argentina de la escala de Monash para la evaluación de la relación de los...

https://doi.org/10.1016/S10900233(02)00237-X

Polheber, J. P., \& Matchock, R. L. (2013). The presence of a dog attenuates cortisol and heart rate in the Trier Social Stress Test compared to human friends. Journal of Behavioral Medicine, 37(5), 860-867. https:// doi.org/10.1007/s10865-013-95461

Rehn, T., \& Keeling, L. J. (2016). Measuring dog-owner relationships: Crossing boundaries between animal behaviour and human psychology. Applied Animal Behaviour Science, 183, 1-9. https://doi.org/10.1016/j. applanim.2016.07.003

Rehn, T., Lindholm, U., Keeling, L., \& Forkman, B. (2014). I like my dog, does my dog like me? Applied Animal Behaviour Science, 150, 65-73. https://doi.org/10.1016/j. applanim.2013.10.008

Rohlf, V. I., Bennett, P. C., Toukhsati, S., \& Coleman, G. (2012). Beliefs underlying dog owners' health care behaviors: Results from a large, self-selected, internet sample. Anthrozoos, 25(2), 171-185. https://doi.org/10.2752/17530371 2X13316289505341

Sheppard, G., \& Mills, D. (2002). The Development of a Psychometric Scale for the Evaluation of the Emotional Predispositions of Pet Dogs Journal Issue. International Journal of Comparative Psychology, 15, 201-222. Retrieved from papers://eba95c07-21c64247-a2f7-57339f1ca15a/Paper/ p11868

Tabachnick, B.G., \& Fidell, L.S. (2013). Using Multivariante Statistics, $6^{\text {th }}$ Edition, Pearson.

Thorn, P., Howell, T. J., Brown, C., \& Bennett, P. C. (2015). The canine cuteness effect: Owner-perceived cuteness as a predictor of human-dog relationship quality. Anthrozoos, 28(4), 569-585. https://doi.org/10.1 080/08927936.2015.1069992

Van Herwijnen, I. R., Van Der Borg, J. A. M., Naguib, M., \& Beerda, B. (2018). The existence of parenting styles in the owner-dog relationship. PLoS ONE, 13(2), 1-14. https://doi. org/10.1371/journal.pone.0193471 